\title{
Integrating radiation oncology into the management of pancreatic cancer
}

\author{
Robert Jäger (D) - Rocco Weigel · Britta Forthuber · Ute Ganswindt
}

Received: 19 December 2018 / Accepted: 26 February 2019 / Published online: 12 March 2019

(C) The Author(s) 2019

\begin{abstract}
Summary Although some improvements have been made in recent years, the prognosis of pancreatic ductal adenocarcinoma remains poor. Surgical resection followed by adjuvant systemic therapy is the only curative treatment option in early stage disease. The role of radiotherapy in the treatment of pancreatic cancer is not well defined and still controversially discussed. Following the results of the ESPAC-1 trial, adjuvant radiochemotherapy (RCT) was no longer employed in most European countries. Nevertheless, in high-risk situations for local recurrence, the addition of adjuvant radiochemotherapy to adjuvant systemic therapy should be discussed, as it may lead to prolonged local tumor control. In resectable tumors, neoadjuvant radiochemotherapy or stereotactic body radiation therapy combined with systemic therapy showed encouraging results in phase I/II trials without increasing postoperative morbidity. Until the results of prospective randomized trials are available, neoadjuvant therapy in resectable pancreatic cancer is only recommended in clinical trials. In borderline resectable and locally advanced tumors, the addition of radiochemotherapy to systemic therapy leads to improved tumor response, and $20-30 \%$ of locally advanced tumors can be resected after neoadjuvant therapy. In locally advanced tumors with stable disease after systemic therapy, the addition of radiochemotherapy should be discussed to increase local control and prolong time to local progression. Modern radiotherapy with image guidance, intensitymodulated radiotherapy, and stereotactic body radiotherapy offer new perspectives for the future and will
\end{abstract}

R. Jäger $(\bowtie) \cdot$ R. Weigel $\cdot$ B. Forthuber $\cdot$ U. Ganswindt Department of Therapeutic Radiology and Oncology, Innsbruck Medical University, Innsbruck, Austria robert.h.jaeger@i-med.ac.at be part of modern multimodal treatment concepts to improve the outcome of pancreatic cancer.

Keywords Pancreatic ductal adenocarcinoma - Neoadjuvant therapy - Radiotherapy - Chemoradiation . Stereotactic body radiation therapy

\section{Introduction}

Pancreatic ductal adenocarcinoma is one of the most challenging diseases of the twenty-first century and is still associated with a fatal prognosis. With a survival expectancy of $5-10 \%$ at 5 years, the prognosis has not improved over the past 10 years. $80 \%$ of the patients are diagnosed with locally advanced disease or metastasis. Only approximately $20 \%$ of the patients present with potentially curable (resectable/ borderline resectable) disease. Standard treatment for resectable disease is upfront surgery followed by adjuvant systemic therapy. Even if the multidisciplinary approach has made some progress, 5 -year overall survival (OS) rates only reach $25-30 \%$ at best [1]. The role of radiotherapy in the treatment of pancreatic cancer is controversially discussed and not yet clearly defined. While the National Comprehensive Cancer Network (NCCN) recommends radio(chemo)therapy more widely, European guidelines limit the use of radiotherapy to the borderline resectable situation after chemotherapy, in clinical trials, or in the palliative setting [2, 3]. Which patients benefit most from radiotherapy cannot yet be fully answered. The aim of this short review is to summarize possibilities and limitations of modern radiotherapy in the treatment of pancreatic cancer as well as to give a future perspective. 


\section{Adjuvant therapy}

5-year survival following surgery with curative intent is less than $20 \%$. Many efforts involving adjuvant chemotherapy, radiotherapy, or the combination of both have been made to improve survival. Adjuvant chemotherapy with gemcitabine was a long-time standard, reaching a 5-year overall survival of 24 months. The results of the ESPAC-4 trial suggest that the addition of capecitabine to gemcitabine results in improved survival [4]. That adjuvant chemotherapy with mFOLFIRINOX is superior to gemcitabine with significantly improved disease-free, metastasis-free, and overall survival has been recently published [5]. The GITSG 9173 trial compared postoperative radiotherapy combined with 5-FU to no adjuvant treatment for resected adenocarcinoma of the pancreas. Patients who received postoperative radiochemotherapy (RCT) had improved survival (20 vs. 11 months median survival) [6]. Referring to this trial, adjuvant RCT is still an acceptable choice for adjuvant treatment of pancreatic cancer in the US.

Most data comparing adjuvant RCT and chemotherapy did not show a clear advantage of the addition of radiation therapy. Results of ESPAC-1 even suggest that the addition of radiation to adjuvant 5-FU chemotherapy may be harmful [7]. We learn from the ESPAC-4 results that there is still a need for improvement of local control after curative resection of pancreatic cancer: $50 \%$ of the patients suffer from local recurrence, and R1-resected patients did not show a clear improvement of median overall survival (23.0 vs. 23.7 months) through the addition of capecitabine compared to R0-resected patients (27.9 vs. 39.5 months) [4]. The EORTC-4001322012/FFCD-9203/GERCOR phase II study randomly assigned patients to receive either four cycles of gemcitabine (control arm) or gemcitabine for two cycles followed by weekly gemcitabine with concurrent radiation (50.4 Gy; $1.8 \mathrm{~Gy}$ per fraction; RCT arm). Median OS was 24 months in both arms. The rate of local recurrence alone as first progression was notably lower in the CRT arm (11 vs. 24\%). The authors concluded that adjuvant gemcitabine-based RCT is feasible, well-tolerated, and not deleterious. Further adding RCT to full-dose adjuvant gemcitabine after resection of pancreatic cancer should be evaluated in a phase III trial [8]. A multi-institutional retrospective review of 955 patients with resected pancreatic cancer evaluated the use of adjuvant RCT (50.4 Gy; $1.8 \mathrm{~Gy}$ per fraction) in combination with 5 -FU or capecitabine chemotherapy compared to adjuvant chemotherapy alone. The results of this analysis showed improved survival with adjuvant RCT compared to chemotherapy alone (median overall survival 39.9 vs. 24.8 months) [9]. Another analysis of the National Cancer Data Base observed superior survival among patients with resected pancreatic adenocarcinoma treated with adjuvant RCT versus adjuvant chemotherapy alone (median overall survival 22.3 vs. 20.0 months). This benefit was pronounced for patients with risk factors for locoregional recurrence, including node positivity and microscopically positive resection margins [10].

In conclusion, the role of adjuvant RCT remains controversial and needs further investigation to clearly define which patients benefit from the addition of RCT to adjuvant chemotherapy. Modern radiotherapy concepts such as stereotactic body radiotherapy (SBRT), intensity-modulated radiotherapy (IMRT), and simultaneous integrated boost (SIB) concepts offer the possibility to apply higher radiation doses to the tumor bed. Whether the combination of these radiation concepts to adjuvant chemotherapy leads to improved survival outcome needs to be validated by randomized trials in the future. The general use of adjuvant RCT cannot be supported according to the present data. Individual therapy concepts including the addition of radiochemotherapy to adjuvant chemotherapy especially for patients with high-risk criteria for locoregional recurrence such as $\mathrm{R} 1$ resection and positive nodal status should be discussed in interdisciplinary tumor boards and may lead to improved local tumor control.

\section{Neoadjuvant therapy}

Due to the relation of the pancreas to adjacent organs and vessels, even patients with resectable pancreatic cancer show a very high rate of $\mathrm{R} 1$ resections, especially at retroperitoneal margins. The implementation of a standard pathological protocol showed an increased $\mathrm{R} 1$ resection rate up to $76 \%$ [11]. In addition, CONKO-001 as well as ESPAC-4 showed that the rate of patients receiving full-dose adjuvant therapy does not exceed $65 \%[4,12]$. The potential benefits of neoadjuvant therapy include a higher proportion of patients with resectable disease experiencing an R0 resection as well as receiving full-dose systemic therapy. Furthermore, down-sizing of the tumor as well as early treatment of micrometastasis could be achieved by the neoadjuvant approach. However, patients with resectable disease receiving neoadjuvant treatment may develop complications which could delay surgery, lead to tumor progression, and even carry the risk of becoming unresectable.

\section{Neoadjuvant radiochemotherapy in resectable pancreatic cancer}

The role of neoadjuvant RCT in resectable pancreatic cancer has been investigated in many clinical trials. Several prospective trials comparing neoadjuvant RCT and surgery to surgery alone showed that neoadjuvant RCT is feasible, safe, and efficacious. However, as a result of slow recruitment and early termination, only non-significant results were obtained $[13,14]$. The recently published GEMCAD 10- 
03 trial studied the use of neoadjuvant gemcitabine/ erlotinib followed by neoadjuvant concomitant RCT (45 Gy; $1.8 \mathrm{~Gy}$ per fraction) with gemcitabine/erlotinib. Adverse effects were limited to mild gastrointestinal disorders. The resectability rate was $76 \%$, with an R0 rate of $63 \%$. R0-resected patients had a better median overall survival compared to R1 or nonresected patients. With a promising median overall survival of 23.4 months, the authors support further investigation in neoadjuvant strategies in resectable pancreatic cancer [15]. A meta-analysis and systematic review were conducted to determine overall survival, mortality rate, and complete resection rate after neoadjuvant RCT compared to resection alone in patients with resectable pancreatic cancer. The analysis revealed that the neoadjuvant group may have an overall survival benefit as compared to the resection alone group, although it did not reach statistical significance. No difference in the in-hospital mortality rate was found. The complete resection rate was significantly higher in the neo-adjuvant group than in the surgery alone group $(p=0.012)$ [16]. Clinical trials such as the phase III NEOPA trial (NCT01900327), which is comparing neoadjuvant gemcitabine RCT to upfront surgery, are still recruiting [17]. According to the available data, neoadjuvant RCT probably leads to higher $\mathrm{R} 0$ resection rates in resectable pancreatic cancer but cannot yet be recommended out of clinical trials.

\section{Neoadjuvant radiochemotherapy in borderline re- sectable pancreatic cancer}

Compared to resectable tumors, borderline resectable tumors are generally amenable for resection but bear an increased risk of incomplete resection and thus poor prognosis. Small retrospective studies and two meta-analyses including patients with resectable and borderline resectable lesions have reported an even better survival outcome after neoadjuvant treatment for these patients than for those with immediately resectable tumors [18, 19]. More recent studies combined induction chemotherapy followed by RCT to improve the results of neoadjuvant treatment. Katz et al. reported on 84 patients treated at the MD Anderson Cancer Center with 2-4 months of gemcitabine-based chemotherapy followed by RCT $(50.4 \mathrm{~Gy}$ in 28 fractions or $30 \mathrm{~Gy}$ in 10 fractions with concomitant 5-FU, paclitaxel, gemcitabine, or capecitabine). 32 patients (38\%) underwent resection and showed negative margins in $96.8 \%$. Median overall survival in resected patients was 40 months versus 15 months in unresected patients $(p=0.001)[20]$. Another group investigated induction FOLFIRINOX followed by chemoradiation (50.4 Gy; 1.8 Gy per fraction) with concomitant gemcitabine or capecitabine in patients suffering from borderline resectable pancreatic cancer. 15 of 18 patients underwent surgery, with 3 found to have occult metastatic disease intraoperatively; 12 (80\%) success- fully underwent R0 resection. FOLFIRINOX followed by RCT was found to be safe and did not adversely affect completion of all intended therapy, including chemoradiation and surgery. In addition, resection rates were favorable and final pathology results were encouraging in this high-risk population [21]. The Alliance A021101 trial evaluated patients with borderline resectable pancreatic cancer. These patients received modified FOLFIRINOX and chemoradiation (50.4 Gy in 28 fractions) with concurrent capecitabine $\left(825 \mathrm{mg} / \mathrm{m}^{2}\right.$, twice a day) prior to intended surgical resection and postoperative gemcitabine $\left(1000 \mathrm{mg} / \mathrm{m}^{2}\right.$ on days 1, 8, and 15 for two cycles). Among 22 patients who started protocol therapy, all 22 completed FOLFIRINOX and 21 (95\%) completed chemoradiation. Among the 15 (68\%) patients who underwent pancreatectomy, 14 (93\%) had negative margins. The patients who underwent resection had an 18-month overall survival rate of $67 \%$ versus $43 \%$ for those who had not undergone resection $(p=0.001)$. Median survival of the overall group was 21.7 months [22]. The ongoing ALLIANCE trial A021501 is investigating preoperative extended chemotherapy (eight cycles of mFOLFIRINOX) versus chemotherapy (seven cycles of mFOLFIRINOX) followed by stereotactic body radiation therapy (SBRT; 33-40Gy in 5 fractions) in patients with borderline resectable pancreatic cancer [23]. The first results of the PREOPANC study group were presented at ASCO 2018. The study recruited 246 patients with resectable or borderline resectable pancreatic cancer. Patients were randomized either to immediate surgery or to preoperative chemotherapy (gemcitabine) and chemoradiotherapy (36 Gy in 15 fractions of $2.4 \mathrm{~Gy}$ with concomitant gemcitabine) followed by surgery. The resection rate in the immediate surgery group was non-significantly increased compared to the group which received preoperative therapy $(72 \%$ vs. $61 \% ; p=0.087)$. However, there was a significant improvement in the R0 resection rate in the neoadjuvant group (63\% vs. $31 \%$; $p=0.001$ ). The reported adverse events were not significantly different. There was an improved survival in the intention to treat analysis, with 17.1 months in the neoadjuvant group compared to 13.7 months in the immediate surgery group, which didn't reach the level of significance $(p=0.074)$. Median diseasefree survival was significantly better in the neoadjuvant group, with 9.9 months versus 7.9 months $(p=0.023)$. The median distant metastasis-free interval was 18.4 months in the neoadjuvant arm versus 10.6 months in the immediate surgery $\operatorname{arm}(p=0.013)$. Additionally, the locoregional recurrence-free interval was improved with neoadjuvant therapy compared to surgery and adjuvant therapy (median: not reached vs. 11.8 months; $p<0.001$ ). A subgroup analysis of patients who actually underwent resection and started adjuvant gemcitabine was performed and showed a median overall survival of 19.1 months in the immediate surgery arm versus 42.1 months in the 
neoadjuvant treatment arm $(p<0.001)$ [24]. According to the favorable results of neoadjuvant therapy in borderline resectable pancreatic cancer, upfront resection is no longer recommended by the NCCN Guidelines [2]. As long as results from randomized trials are still lacking, patients with borderline resectable lesions should be included in clinical trials. In routine practice, if a patient is not included in a trial, a period of chemotherapy (gemcitabine or FOLFIRINOX) followed by RCT and surgery is recommended by the EMSO practical guidelines [3].

\section{Treatment of locally advanced disease}

About one third of the patients with pancreatic carcinoma present with unresectable tumors but without distant metastasis. These tumors are defined as locally advanced disease and are commonly considered as incurable. The treatment of locally advanced tumors has been evaluated in many trials and consists of either systemic therapy alone or in combination with RCT. Regardless of the treatment strategy, median overall survival remains poor, with 12-15 months. The advantage of RCT over best supportive care was evaluated in a small randomized trial and showed significantly improved overall survival, better quality of life, and a lower rate of distant metastasis in the RCT group [25]. The GITSG trial demonstrated the superiority of 5-FU based RCT over radiotherapy alone in locally advanced pancreatic cancer. In the RCT group, mean overall survival was significantly better than in the radiotherapy arm with 10.4 vs. 6.3 months. The trial further showed that a dose escalation of $60 \mathrm{~Gy}$ compared to $40 \mathrm{~Gy}$ did not improve survival but caused higher treatment-related toxicity [26]. Three prospective randomized trials have been conducted comparing the use of RCT to chemotherapy alone in locally advanced pancreatic cancer. In the FFCD/SFRO phase III trial, 119 patients with locally advanced tumors were randomly assigned to either induction chemoradiotherapy (60Gy; $2 \mathrm{~Gy}$ per fraction, combined with 5 -FU/cisplatin) or gemcitabine without radiotherapy. Both arms received maintenance gemcitabine up to disease progression or severe treatment-related toxicity. Overall survival was lower in the RCT group, with 8.6 months compared to 13 months in the chemotherapy group. In must be acknowledged that in the RCT group, only $42 \%$ of the patients received at least $75 \%$ of the scheduled radiation and chemotherapy dose [27]. In another trial comparing RCT $(50.4 \mathrm{~Gy} ; 1.8 \mathrm{~Gy}$ per fraction) with concomitant gemcitabine to gemcitabine alone, overall survival was significantly improved in the chemoradiation arm (11.1 vs. 9.2 months). Gemcitabine in combination with radiotherapy caused increased but manageable toxicities compared to chemotherapy alone [28]. The LAP07 randomized trial investigated the effect of chemoradiotherapy $(54 \mathrm{~Gy}$; $1.8 \mathrm{~Gy}$ per fraction with concurrent capecitabine) ver- sus chemotherapy alone on survival in patients with locally advanced pancreatic cancer, controlled after 4 months of gemcitabine with or without erlotinib. Median overall survival was not significantly different between chemotherapy with 16.5 months and RCT with 15.2 months. RCT was associated with decreased local progression and didn't show an increase in grade 3 to 4 toxicity, except for nausea. The median delay until treatment reinduction was significantly longer in the RCT group, with 6.1 months compared to 3.7 months in the chemotherapy group $(p=0.02)$ [29]. A systematic review and meta-analysis of response and resection percentages after neoadjuvant therapy showed that approximately one third of initially nonresectable pancreatic tumors became resectable after neoadjuvant therapy. Survival was comparable with initially resected patients [18]. These results could be confirmed by Habermehl et al. After neoadjuvant RCT (52.2 Gy; $1.8 \mathrm{~Gy}$ per fraction) with concomitant gemcitabine, $26 \%$ of initially unresectable locally advanced pancreatic cancers were able to undergo secondary resection. Clear margins could be achieved in $39.2 \%$. Patients with complete resection after RCT showed significantly increased median overall survival of 22.1 compared to 11.9 months in non-resected patients [30]. A recently published retrospective analysis evaluated the additional use of preoperative RCT after induction FOLFIRINOX for borderline or locally advanced pancreatic adenocarcinoma. Patients with additional RCT had significantly higher R0 resection rates $(89.2 \%$ vs. $76.3 \% ; p=0.017)$, ypN0 rates $(76.2 \%$ vs. $48.5 \% ; p<0.001)$, as well as higher rates of pathologic major response $(33.3 \%$ vs. $12.9 \% ; p=0.001)$. Patients receiving FOLFIRINOX and RCT had a lower rate of locoregional relapse $(28.3 \%$ vs. $50.7 \% ; p=0.004)$ and significantly longer overall survival (57.8 vs. 35.5 months; $p=0.007$ ) [31]. The role of RCT in the treatment of locally advanced tumors is still controversially discussed. Ongoing prospective randomized trials such as the ABCSG P02 trial, investigating the additional use of RCT after induction FOLFIRINOX in locally advanced pancreatic cancer, should be supported. Patients with locally advanced unresectable tumors showing no signs of tumor progression after 3 cycles of chemotherapy should be evaluated for RCT and secondary resection.

New concepts: intensity-modulated radiation therapy, image guidance, dose escalation, and stereotactic body radiotherapy

Improvement of local tumor control without impairing the tolerability of treatment has been one of the core interests of modern radiotherapy in recent years. The use of more modern radiation therapy procedures, such as contrast-enhanced $4 \mathrm{D}$ computed tomography and intensity-modulated radiation therapy (IMRT), offers the possibility of applying higher radiation doses without increasing treatment-related tox- 
Fig. 1 Simultaneous integrated boost (SIB) Concept: PTV SIB (area within the yellow isodose) receives 54 Gy (2.16Gy/fraction) while the PTV adjacent to organs at risk (area within the red isodose) receives $45 \mathrm{~Gy}$ (1.8Gy/fraction). Gy Gray

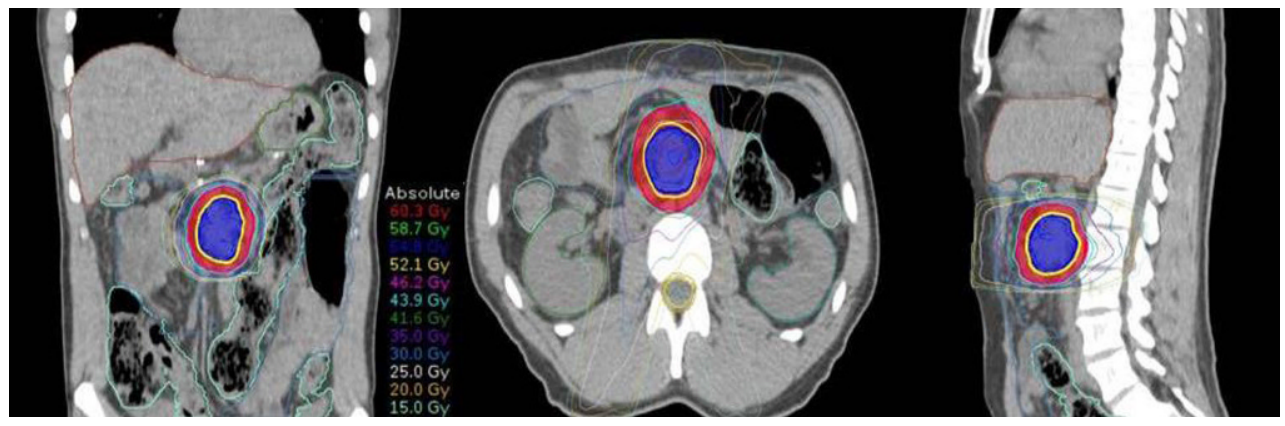

icities. Prospective trials comparing IMRT to conventional 3D radiotherapy are not yet published for pancreatic cancer. In a systematic review, authors compared treatment-related toxicities from IMRT and 3D trials. Although no substantial difference in survival was observed between the two treatment techniques, significantly lower grade 3 and higher acute and late toxicities for IMRT have been reported [32]. Due to limited tolerance of adjacent normal structures such as the small bowel and the stomach, the standard radiation dose in the treatment of pancreatic cancer was limited to $50.4 \mathrm{~Gy}$. However, with IMRT, the total dose to the tumor can be increased while respecting the dose constraints of normal tissues. The MD Anderson Cancer Center group recently published the results of a retrospective cohort study of patients with biopsy-proven locally advanced pancreatic cancer treated with definitive chemoradiation. Improved outcomes in terms of overall survival and locoregional relapse-free survival were demonstrated in patients who received an escalated dose (biological equivalent dose [BED] >70 Gy) compared to those with the standard radiation dose or biological equivalent doses of $<70$ Gy. There were no differences in toxicity between the groups receiving BED $<70 \mathrm{~Gy}$ or $>70 \mathrm{~Gy}$ [33]. Another advantage of modern radiotherapy techniques is the ability to apply higher dose levels at certain areas with an increased risk of local failure, so-called simultaneously integrated boost (SIB) concepts (see Fig. 1). Surgical clips may help to identify areas with an increased risk of local failure and can be used for image guidance during radiotherapy but are not yet standard. The application of surgical clips should be preoperatively discussed with the radio-oncologist as there are many different possibilities and materials. Another possibility of local dose escalation is the application of intraoperative radiation therapy (IORT) to the tumor bed during surgery. IORT has the advantage of enabling the delivery of high doses to areas at risk for microscopic disease, while sparing nearby dose-limiting organs at risk. IORT is typically used as a boost after preoperative or prior to postoperative external beam radiotherapy (EBRT). IORT trials are mostly single center experiences with small numbers of patients. Krempien et al. summarized the use of IORT in the multidisciplinary management of pancreatic cancer. The applied doses vary from 10-25 Gy and are not only used in resected pancreatic cancer but also in locally advanced/unresectable pancreatic cancer. The authors conclude that IORT is a safe addition to the neoadjuvant and adjuvant treatment of pancreatic cancer, with the intention of improving local control [34].

Many recently published phase I-II trials have explored the benefit of stereotactic body radiation therapy (SBRT) in the treatment of pancreatic cancer, especially in borderline resectable und locally advanced disease. SBRT implements highly conformal and precise delivery of radiotherapy to apply local ablative doses to treatment volumes. SBRT is well established in the treatment of central nervous system lesions as well as pulmonary lesions. Nowadays, improvements in image guidance and patient positioning allow SBRT even to upper abdominal areas. To date, several early phase prospective trials exploring the safety and efficacy of SBRT in the treatment of pancreatic cancer have been conducted. A systemic review and pooled analysis of 19 trials of SBRT for locally advanced pancreatic cancer was recently published by Petrelli et al. The delivered dose ranged from 18 to $50 \mathrm{~Gy}$ in 1 to 8 fractions, with generally acceptable grade 3 to 4 toxicities [35]. The short treatment time offers new interesting possibilities to integrate SBRT in the treatment course of full-dose systemic therapy. However, a definitive validation of this treatment modality in large randomized trials is required. Especially neoadjuvant concepts with short-course SBRT followed by full-dose systemic therapy and resection are probably one of the most interesting treatment strategies for the future.

\section{Treatment-related toxicities}

Treating pancreatic cancer with radio(chemo)therapy is challenging due to the anatomic neighborhood of highly radiosensitive organs such as the small bowel, the kidneys, and the spinal cord. Toxicities vary depending on the dose and the schedule of radiation delivered. The major toxicities reported are related to injury of the duodenum due to the low tolerance of the intestinal mucosa to radiation. Smaller treatment volumes and more conformal dose application (IMRT, VMAT) in combination with image guidance have reduced treatment-related toxicities over the years. An 
important confounding factor for toxicity is the use of concurrent chemotherapeutic agents such as gemcitabine, infusional 5-FU, or capecitabine. Modern radiotherapy allows a combination with systemic therapy, still causing only mild and manageable treatment-related toxicities. Reported adverse toxic events in the LAP07 randomized trial were not different between the chemotherapy and the RCT group except for 6 patients $(5.9 \%)$ in the RCT group who suffered from grade 3 or 4 nausea [29]. Preliminary results of the PREOPANC trial comparing neoadjuvant RCT to immediate surgery for resectable and borderline resectable pancreatic cancer did not show an increased rate of serious adverse events in the RCT arm $(39 \%$ vs. $46 \% ; p=0.28$ ) [24]. That IMRT (median 54Gy) with concurrent capecitabine $\left(1600 \mathrm{mg} / \mathrm{m}^{2}\right.$ daily in two divided doses, 5 days a week) is well tolerated could be demonstrated by Ben-Josef et al. 53\% of the patients suffered from grade $1 / 2$ nausea/vomiting and $60 \%$ of the patients developed grade $1 / 2$ hematologic toxicity. Only 1 patient (7\%) experienced grade 3 toxicity, which consisted of gastric ulceration and bleeding that responded to conservative treatment [36]. A systematic review of SBRT for locally advanced pancreatic cancer reported a generally acceptable rate of acute grade 3 to 4 toxicities. Only 3 out of 19 studies detected grade 3 and 4 gastrointestinal toxicity rates of $>10 \%$. The proportion of chronic (late) grade 3 to 4 events did not exceed $11 \%$ (range $0-11 \%$ ). In six series the grade 3 to 4 toxicity rate was $0 \%$ [35]. In conclusion, modern radiotherapy concepts, even in combination with systemic therapy, are generally well tolerated, and treatment-related toxicities are limited to manageable grade 2-3 toxicities. Nevertheless, high dose radiotherapy may also cause severe adverse events. Therefore, treatment volumes and radiation exposure to organs at risks must be wisely considered, especially when radio-sensitizing systemic agents are concurrently applied.

\section{Conclusion}

The role of radiotherapy in the treatment of pancreatic cancer is still not well defined and has been controversially discussed. Most present guidelines do not recommend standard use of radiochemotherapy out of clinical trials. In the adjuvant setting, individual therapy concepts including the addition of radiochemotherapy to adjuvant chemotherapy for patients with high-risk features for locoregional recurrence, such as $\mathrm{R} 1$ resection and positive nodal status, should be discussed in interdisciplinary tumor boards and may lead to improved local tumor control. In the neoadjuvant setting, RCT and SBRT concepts lead to higher R0 resection rates and better local tumor control. Especially neoadjuvant concepts with short course SBRT followed by full-dose systemic therapy and resection will be one of the most interesting treatment strategies in the future. For borderline resectable disease, outside of clinical studies, a period of chemotherapy (gemcitabine or FOLFIRINOX) followed by RCT and evaluation for surgery is a feasible strategy in routine practice. Patients with locally advanced unresectable tumors showing no sign of tumor progression after three cycles of chemotherapy should be evaluated for RCT and secondary resection. The results of prospective randomized trials are needed to define the role of radiotherapy in the treatment of pancreatic cancer. Especially the evaluation of modern radiotherapy concepts such as IMRT and SBRT in combination with modern systemic agents will hopefully improve outcomes in the future.

Funding Open access funding provided by University of Innsbruck and Medical University of Innsbruck.

Conflict of interest R. Jäger, R. Weigel, B. Forthuber, and U. Ganswindt declare that they have no competing interests.

Open Access This article is distributed under the terms of the Creative Commons Attribution 4.0 International License (http://creativecommons.org/licenses/by/4.0/), which permits unrestricted use, distribution, and reproduction in any medium, provided you give appropriate credit to the original author(s) and the source, provide a link to the Creative Commons license, and indicate if changes were made.

Publisher's Note Springer Nature remains neutral with regard to jurisdictional claims in published maps and institutional affiliations.

\section{References}

1. Katz MH, et al. Long-term survival after multidisciplinary management of resected pancreatic adenocarcinoma. Ann Surg Oncol. 2009;16:836-47.

2. Tempero MA, et al. NCCN clinical practice guideline in oncology pancreatic Adenocarcinoma version 2.2017. J Natl Compr Canc Netw. 2017;15(8):1028-61. https://doi. org/10.6004/jnccn.2017.0131.

3. Ducreux M, et al. Cancer of the pancreas: EMSO clinical practice guidelines for diagnosis, treatment and follow up. Ann Oncol. 2015;26(suppl.5):56-68.

4. Neoptalemos JP, et al. Comparison of adjuvant gemcitabine and capecitabine with gemcitabine monotherapy in patients with resected pancreatic cancer (ESPAC-4): A multicentre, open label, randomised, phase 3 trial. Lancet. 2017;389:1011-24.

5. Conroy T, et al. FOLFIRINOX or Gemcitabine as Adjuvant therapy for pancreatic cancer. N Eng J Med. 2018;379:2395-406.

6. Kalser MH, et al. Pancreatic cancer: Adjuvant combined radiation and chemotherapy following curative resection. Arch Surg. 1985;120:899-903.

7. Neoptalemos JP, et al. A Randomized trial of chemoradiotherapy and chemotherapy after resection of pancreatic cancer. NEng J Med. 2004;350(12):1200-10.

8. Van Laethem JL, et al. Adjuvant gemcitabine alone versus gemcitabine-based chemoradiotherapy after curative resection for pancreatic cancer: A randomized EORTC40013-22012/FFCD-9203/GERCOR Phase II Study. J Clin Oncol. 2010;28(29):4450-6.

9. Morganti AG, et al. Multi-institutional pooled analysis on adjuvant chemoradiation in pancreatic cancer. Int J Radiat OncolBiol Phys. 2014;90(4):911-7. 
10. Rutter CE, et al. Addition of radiotherapy to adjuvant chemotherapy is associated with improved overall survival in resected pancreatic adenocarcinoma: An analysis of the National Cancer Data Base. Cancer. 2015;121(23):4141-8. https://doi.org/10.1002/cncr.29652.

11. Esposito I, et al. Most pancreatic cancer resections are R1 resections. Ann Surg Oncol. 2008;15:1651-60.

12. Oettle $\mathrm{H}$, et al. Adjuvant chemotherapy with gemcitabine and long-term outcomes among patients with resected pancreatic cancer. The CONKO-001 randomized trial. JAMA. 2013;310(14):1473-81.

13. Golcher $\mathrm{H}$, et al. Neoadjuvant chemoradiation therapy with gemcitabine/cisplatin and surgery versus immediate surgery in resectable pancreatic cancer. Strahlenther Onkol. 2015;191:7-16.

14. Casadei R, et al. Neoadjuvant chemoradiotherapy and surgery versus surgery alone in resectable pancreatic cancer: A single-center prospective, randomized, controlled trial which failed to achieve accrual targets. J Gastrointest Surg. 2015;19:1802-12.

15. Maurel J, et al. Outcomes after neoadjuvant treatment with gemcitabine and erlotinib followed by gemcitabineerlotinib and radiotherapy for resectable pancreatic cancer (GEMCAD 10-03 trial). Cancer Chemother Pharmacol. 2018;82:935-43.

16. Wei L, et al. Efficacy of neo-adjuvant chemoradiotherapy for resectable pancreatic adenocarcinoma. A PRISMAcompliant meta-analysis and systemic review. Medicine. 2016;95(15):1-8.

17. Tachezy M, et al. Sequential neoadjuvant chemoradiotherapy (CRT) followed by curative surgery vs. primary surgery alone for resectable, non-metastasized pancreatic adenocarcinoma: NEOPA - a randomized multicenter phase III study (NCT01900327, DRKS00003893, ISRCTN82191749). BMCCancer. 2014;14:411-20.

18. Gillen S, et al. Preoperative/neoadjuvant therapy in pancreatic cancer: A systemic review and meta-analysis of response and resection percentages. Plos Med. 2010;7:e1000267.

19. Assifi MM, et al. Neoadjuvant therapy in pancreatic adenocarcinoma: A meta-analysis of phase II trials. Surgery. 2011;150:466-73.

20. Katz MH, et al. Borderline resectable pancreatic cancer: The importance of this emerging stage of disease. J Am Coll Surg. 2008;206:833-48.

21. ChristiansK, etal. NeoadjuvantFOLFIRINOXfor borderline resectable pancreas cancer; A new treatment paradigm? Oncologist. 2014;19:266-74.

22. Katz MH, et al. Preoperative modified FOLFIRINOX treatment followed by capecitabine-based chemoradiation for borderline resectable pancreatic cancer: Alliance for clinical trials in oncology trial A021101. Jama Surg. 2016;151:e161137.

23. Katz MH, et al. Alliance for clinical trials in oncology (ALLIANCE) trial A021501: Preoperative extended chemotherapy vs. chemotherapy plus hypofractionated radiation therapy for borderline resectable adenocarcinoma of the head of the pancreas. BMCCancer. 2017;17:505-12.
24. Versteijne E, et al. Preoperative chemoradiotherapy potentially improves outcomefor (borderline) resectable pancreatic cancer: Preliminary results of the Dutch randomized phase III PREOPANC trial. Int J Radiat Oncol Biol Phys. 2018;102(5):1606-7.

25. Shinchi $\mathrm{H}$, et al. Length and quality of survival after external beam radiotherapy with concurrent continuous 5 -flourouracil infusion for locally unresectable pancreatic cancer. Int J Radiat Oncol Biol Phys. 2002;53(1):146-50.

26. Moertel CG, et al. Therapy of locally advanced pancreatic carcinoma: A randomized comparison of high dose (6000 rads) radiation alone, moderate dose radiation $(4000$ rads) +5 -flourouracil and high dose radiation +5 fluorouracil: The Gastrointestinal Tumor Study Group. Cancer. 1981;48(8):1705-10.

27. Chauffert B, et al. Phase III trial comparing intensive induction chemoradiotherapy (60 Gy, infusional 5-FU and intermittent cisplatin) followed by maintenance gemcitabine with gemcitabine alone for locally advanced unresectable pancreatic cancer. Definitive results of the 2000-01 FFCD/ SFRO study. Ann Oncol. 2008;19:1592-9.

28. Loehrer PJ, et al. A randomized phase III study of gemcitabine in combination with radiation therapy versus gemcitabine alone in patients with localized, unresectable pancreatic cancer: An Eastern Cooperative Oncology Group trial. JClin Oncol. 2007;25(3):326-31.

29. HammelP, etal. Effect of chemoradiotherapyvs chemotherapy on survival in patients with locally advanced pancreatic cancer controlled after 4 months of gemcitabine with or without erlotinib. The LAP07 Randomized clinical trial. JAMA. 2016;315(17):1844-53.

30. Habermehl D, et al. Neoadjuvant chemoradiation with gemcitabine for locally advanced pancreatic cancer. Radiat Oncol. 2012;7:28-34.

31. Pietrasz D, et al. How does chemoradiotherapy following induction FOFIRINOX improve the results in resected borderline or locally advanced pancreatic adenocarcinoma? An AGEO-FRENCH Multicentric Cohort. Ann Surg Oncol. 2018; https:// doi.org/10.1245/s10434-018-6931-6.

32. Bittner MI, et al. Comparison of toxicity after IMRT and 3Dconfromal radiotherapy for patients with pancreatic cancer-a systemic review. Radiother Oncol. 2015;114:117-21.

33. Krishnan S, et al. Focal radiation therapy dose escalation improves overall survival in locally advanced pancreatic cancer patients receiving induction chemotherapy and consolidative chemoradiation. Int J Radiat Oncol Biol Phys. 2016;94(4):755-65.

34. Krempien R, et al. Intraoperative radiation therapy (IORT) in pancreatic cancer. Radiat Oncol. 2017;12(8) https://doi. org/10.1186/s13014-016-0753-0.

35. Petrelli F, et al. Stereotactic body radiation therapy for locally advanced pancreatic cancer: A Systemic review and pooled analysis of 19 trials. Int J Radiat Oncol Biol Phys. 2017;97(2):313-22.

36. Ben-Josef E, et al. Intensity-modulated Radiotherapy (IMRT) an concurrent capecitabine for pancreatic cancer. Int J Radiat Oncol Biol Phys. 2004;59(2):454-9. 